\title{
Bipolar disorder and addictions: the elephant in the room
}

Paul R. A. Stokes, Nicola J. Kalk and Allan H. Young

\section{Summary}

Addictions are highly prevalent in bipolar disorder and greatly affect clinical outcomes. In this editorial, we review the evidence that addictions are a key challenge in bipolar disorder, examine putative neurobiological mechanisms, and reflect on the limited clinical trial evidence base with suggestions for treatment strategies and further developments.

\section{Declaration of interest}

P.R.A.S. has received support for research, expenses to attend conferences and fees for lecturing and consultancy work (including attending an advisory board) from life sciences companies including corcept Therapeutics, Indivior and Liva Nova. He is a consultant psychiatrist within a tertiary level specialist service and a specialist consultant advisor in mood disorders for the UK Civil Aviation Authority. N.J.K. has received funding for educational activities, including travel expenses, and research expenses during her PhD (2010-2013) from GlaxoSmithKline (GSK), as her PhD was funded by a Wellcome Trust GSK Translational Medicine Training Fellowship. A.H.Y. has been commissioned to give lectures and is on advisory boards for all major pharmaceutical companies with drugs used in affective and related disorders. He was the lead Investigator for Embolden Study (AstraZeneca), BCl Neuroplasticity study and Aripiprazole Mania Study. He has been involved in investigator initiated studies from AstraZeneca, Eli Lilly, Lundbeck and Wyeth. A.H.Y. has been awarded research grants from: National Institute of Mental Health (USA); Canadian Institutes of Health Research (Canada); National Association for Research on Schizophrenia And Depression (USA); Stanley Medical Research Institute (USA); Medical Research Council (UK); Wellcome Trust (UK); Royal College of Physicians (Edinburgh, UK); British Medical Association (UK); UBC-VGH Foundation (Canada); Western Economic Development Council (Canada); Coast Capital Savings Depression Research Fund (Canada); Michael Smith Foundation for Health Research (Canada); National Institute for Health Research (NIHR) (UK).

\section{Copyright and usage}

(c) The Royal College of Psychiatrists 2017.
Paul R. A. Stokes (pictured) is a Clinical Senior Lecturer in Mood Disorders at the Centre for Affective Disorders, Institute of Psychiatry, Psychology and Neuroscience, King's College London and a Consultant Psychiatrist with the National Affective Disorders Service, South London and Maudsley NHS Foundation Trust. His research interests include the use of neuroimaging to better understand and treat bipolar disorder and related comorbid addictions Nicola J. Kalk is an Academic Clinical Lecturer at the National Addictions Centre at King's College London and works clinically in the Centre for Affective Disorders. Her research interests include inflammatory, endocrine and neurochemical pathways which contribute to the pathophysiology of alcohol dependence, mood disorders, and their comorbidity. Allan $\mathrm{H}$. Young holds the Chair of Mood Disorders at King's College London where he also is Director of the Centre for Affective Disorders.

Bipolar disorder is one of the most challenging mental health disorders to assess and treat and a key challenge is the very high rates of addiction comorbidities experienced by people with bipolar disorder. The increased prevalence of addictions in bipolar disorder is demonstrated by the recent United States National Epidemiologic Survey on Alcohol and Related Conditions which found that people with bipolar I disorder have a 5.8 times increased lifetime risk of a substance use disorder diagnosis according to DSM-V criteria. This risk was still 2.3 times increased even after adjusting for other psychiatric comorbidities. ${ }^{1}$ Studies in the UK reflect the increased risk of addictions in bipolar disorder, with $48 \%$ and $44 \%$ of people with bipolar disorder experiencing a lifetime history of alcohol and substance misuse respectively. ${ }^{2}$ Bipolar disorder has one of the highest rates of cigarette smoking of any mental health disorder and the lowest rate of smoking cessation. ${ }^{3}$ Increased rates of behavioural addictions, such as gambling disorder, ${ }^{4}$ have also been identified in bipolar disorder and approximately 1 in 10 people with bipolar disorder may have a moderate to severe lifetime risk of problem gambling. ${ }^{4}$ Addiction comorbidity in bipolar disorder is important as it greatly affects clinical outcomes. For example, addiction comorbidity in bipolar disorder is associated with more severe manic episodes, and increased risks of violence, suicide and relapse. ${ }^{5,6}$ Despite their greater needs and increased risk, people with bipolar disorder and addiction comorbidity often struggle to access secondary mental health services. ${ }^{7}$

\section{Why are addictions so prevalent in bipolar disorder?}

The association between bipolar disorder and addictions raises the question: why are people with bipolar disorder at increased risk of addictions? There are several potential mechanisms. The first is that people with bipolar disorder 'self-medicate' with alcohol or drugs to alleviate mood episodes. However, only $25 \%$ of people with bipolar disorder increase their use of alcohol during a manic episode and most do not change their use during a depressive episode. ${ }^{8}$ Indeed, people with bipolar disorder and addiction comorbidities are no different to people with substance use disorder in experiencing increased substance sensitivity or sensation seeking, and in using substances to achieve a sense of euphoria or to alleviate mood and anxiety symptoms irrespective of current mood episode. They also report similar motivations for substance use as the general population. ${ }^{9}$

The second potential mechanism is a shared neurobiology between bipolar disorder and addictions, which means that people with bipolar disorder are at risk of addictions. Indeed, the prospective Zurich cohort study showed that manic symptoms or a diagnosis of bipolar II disorder was associated with a greatly increased risk of developing alcohol or benzodiazepine dependence over 20 years. ${ }^{10}$ Certainly there is evidence that young 
men who report high rates of hypomanic symptoms, and may be at risk of developing bipolar disorder, experience similar low-level behavioural responses to alcohol as is found in those with a family history of alcohol dependence. ${ }^{11}$

Addiction comorbidities in bipolar disorder are likely to be mediated by the involvement of a number of neurotransmitter systems. Much of the focus of addiction research has been on the dopamine neurotransmitter system and it is likely that vulnerability to addictions in bipolar disorder may be mediated, at least in part, through dopaminergic dysregulation. However, the evidence for this is limited by a lack of studies of the dopamine system in people with bipolar disorder and addiction comorbidity. ${ }^{12}$ Anxiety disorders are also highly prevalent in bipolar disorder and so there is also the possibility that people with bipolar disorder use alcohol or other substances to alleviate anxiety symptoms perhaps generated by gamma- aminobutyric acid-A receptor dysregulation previously reported in anxiety disorders. ${ }^{13}$

\section{Treatment approaches}

How to do we best treat people with bipolar disorder and comorbid addictions? The first step is to ensure that comorbid addictions are adequately identified in people with bipolar disorder. National Institute for Health and Care Excellence guidelines recommend that all patients who disclose substance use are asked about quantity, frequency and pattern of use, route of administration and duration of current level of use. ${ }^{14}$ It is also important to establish whether criteria for substance dependence are met as this will greatly influence clinical management. ${ }^{14}$ We would suggest that a history of behavioural addictions such as gambling, sexual and shopping addictions is also taken. Frequent reassessment of substance use and behavioural addictions is recommended especially following a change in mental state. ${ }^{15}$ Once addiction comorbidity has been identified, the intensity of intervention will depend on the severity of the problem identified and resources available locally, but joint working with expert addictions services is recommended to achieve the best outcomes. ${ }^{7}$ It is important to note that addiction services are rarely commissioned to provide psychiatric care and so secondary care mental health services should lead treatment and people with bipolar disorder should not be excluded from these services because of addiction comorbidity. ${ }^{7}$ Clinicians should also develop care plans that consider the potential for greater mood instability, higher risks of self-harm and poorer medication adherence found in people with bipolar disorder with addictions comorbidities.

\section{Pharmacological management}

The pharmacological management of bipolar disorder with addiction comorbidities is complicated by a lack of good-quality, placebo-controlled, randomised control trials (RCTs). This may partly be due to pharmaceutical industry sponsored trials excluding people with bipolar disorder and comorbid addictions. The evidence that does exist is based on a relatively small number of RCTs which typically assess the effects of interventions in less than 30 participants over a 12 -week period. Several RCTs have investigated the effects of mood stabilisers on substance use and mood scores. Although we are not aware of any studies that have examined the effect of lithium in people with bipolar disorder and alcohol dependence, a large multicentre trial of lithium in alcohol-dependent people with a history of depression found no improvement in alcohol consumption or mood scores. ${ }^{16}$ Another study found that sodium valproate in addition to lithium and psychosocial interventions improved alcohol consumption but not mood scores in people with bipolar disorder and alcohol dependence. ${ }^{17}$ Turning to bipolar disorder with substance use disorder comorbidity, a large trial that compared the effectiveness of lithium and valproate compared with lithium alone failed due to lack of adherence and non-response to acute treatment. ${ }^{18}$ Lamotrigine has been found to improve the amount spent on cocaine but not mood scores in people with bipolar disorder and cocaine dependence ${ }^{19}$ and, in a small RCT, lithium has been found to be effective in reducing gambling behaviour and mood instability in pathological gamblers with bipolar disorder. $^{20}$

There are surprisingly few studies of the effectiveness of antipsychotic medication in people with bipolar disorder and comorbid addictions. Those studies that are available have examined the effectiveness of adding quetiapine to mood stabilisers for alcohol use disorder comorbidity and have shown that it is largely ineffective in improving either alcohol consumption or mood measures. ${ }^{21,22}$

Two small pilot RCTs have investigated the effectiveness of adjunctive acamprosate or naltrexone in alcohol-dependent people with bipolar disorder. Neither found improvements in alcohol consumption or mood scores, ${ }^{23,24}$ although naltrexone was associated at a trend significance level with fewer drinking days and lower craving scores, and both medications were safe, well-tolerated and did not destabilise mood. Naltrexone is recommended by the British Association for Psychopharmacology treatment guidelines to help people with bipolar disorder to reduce their alcohol consumption and acamprosate is then recommended if naltrexone has not been effective in supporting abstinence. $^{15}$

\section{Limitations}

There are several limitations which constrain our understanding of addiction comorbidity in bipolar disorder and how best to provide treatment. Most of the prevalence data originate from high-income countries, particularly the USA, and there is a real need for comorbidity prevalence data from low- and middleincome countries. Given the importance of addictions in bipolar disorder, there are also surprisingly few studies which have investigated neurobiological mechanisms which mediate addictions comorbidity. There have also been few well-powered RCTs which have investigated the effectiveness of pharmacological treatments for bipolar disorder with addiction comorbidity and the lack of an evidence base is a real challenge in determining best treatment practice.

\section{Conclusions}

Addictions are highly prevalent in bipolar disorder and affect clinical outcomes and risk profiles. The reasons why people with bipolar disorder are at high risk of addiction remain unknown and require further investigation but may be mediated by shared neurobiological mechanisms. Treating addictions in bipolar disorder requires an inclusive, comprehensive approach which supports the identification of addiction comorbidity in bipolar disorder and the development of care plans to minimise risks. There is an urgent need for well-powered placebo-controlled RCTs in this area and, as addictions are so prevalent in bipolar disorder, we suggest that excluding people with bipolar disorder with a history of addictions from clinical trials significantly limits the generalisability of findings to the 'real life' bipolar disorder population. 
Paul R. A. Stokes, PhD, Nicola J. Kalk, PhD, Allan H. Young, FRCPsych, Centre for Affective Disorders, Department of Psychological Medicine, Institute of Psychiatry, Psychology and Neuroscience, King's College London, London, UK

Correspondence: Dr Paul Stokes, Clinical Senior Lecturer in Mood Disorders, Centre for Affective Disorders, Institute of Psychiatry, Psychology \& Neuroscience (IOPPN), Room E2.06, PO72 De Crespigny Park, Denmark Hill, London SE5 8AF, UK. Email: paul.r.stokes@kcl.ac.uk

First received 20 Dec 2016, final revision 4 Apr 2017, accepted 8 May 2017

\section{Funding}

This report represents independent research funded by the NIHR Biomedical Research Centre at South London and Maudsley NHS Foundation Trust and King's College London. The views expressed are those of the authors and not necessarily those of the National Health Service, the NIHR or the Department of Health.

\section{References}

1 Blanco C, Compton WM, Saha TD, Goldstein BI, Ruan WJ, Huang B, et al Epidemiology of DSM-5 bipolar I disorder: results from the National Epidemiologic Survey on Alcohol and Related Conditions - III. J Psychiatr Res 2017; 84: 310-7.

2 Cassidy F, Ahearn EP, Carroll BJ. Substance abuse in bipolar disorder. Bipolar Disord 2001; 3: 181-8.

3 Lasser K, Boyd JW, Woolhandler S, Himmelstein DU, McCormick D, Bor DH Smoking and mental illness: a population-based prevalence study. JAMA 2000; 284: 2606-10.

4 Jones L, Metcalf A, Gordon-Smith K, Forty L, Perry A, Lloyd J, et al. Gambling problems in bipolar disorder in the UK: prevalence and distribution. Br J Psychiatry 2015; 207: 328-33.

5 Salloum IM, Cornelius JR, Mezzich JE, Kirisci L. Impact of concurrent alcoho misuse on symptom presentation of acute mania at initial evaluation. Bipolar Disord 2002; 4: 418-21.

6 Dalton EJ, Cate-Carter TD, Mundo E, Parikh SV, Kennedy JL. Suicide risk in bipolar patients: the role of co-morbid substance use disorders. Bipolar Disord 2003; 5: 58-61.

7 National Institute for Health and Care Excellence. Coexisting Severe Mental IIIness and Substance Misuse: Community Health and Social Care Services. NICE, 2016.

8 Strakowski SM, DelBello MP, Fleck DE, Arndt S. The impact of substance abuse on the course of bipolar disorder. Biol Psychiatry 2000; 48: 477-85.

9 Bizzarri JV, Rucci P, Sbrana A, Gonnelli C, Massei GJ, Ravani L, et al. Reasons for substance use and vulnerability factors in patients with substance use disorder and anxiety or mood disorders. Addict Behav 2007; 32: 384-91.

10 Merikangas KR, Herrell R, Swendsen J, Rossler W, Ajdacic-Gross V, Angst J. Specificity of bipolar spectrum conditions in the comorbidity of mood and substance use disorders: results from the Zurich cohort study. Arch Gen Psychiatry 2008; 65: 47-52.
11 Yip SW, Doherty J, Wakeley J, Saunders K, Tzagarakis C, de Wit H, et al. Reduced subjective response to acute ethanol administration among young men with a broad bipolar phenotype. Neuropsychopharmacology 2012; 37: 1808-15.

12 Nutt DJ, Lingford-Hughes A, Erritzoe D, Stokes PR. The dopamine theory of addiction: 40 years of highs and lows. Nat Rev Neurosci 2015; 16: 305-12.

13 Nikolaus S, Antke C, Beu M, Muller HW. Cortical GABA, striatal dopamine and midbrain serotonin as the key players in compulsive and anxiety disordersresults from in vivo imaging studies. Rev Neurosci 2010; 21: 119-39.

14 National Institute for Health and Care Excellence. Coexisting Severe Mental IIIness (Psychosis) and Substance Misuse: Assessment and Management in Healthcare Settings (Clinical Guideline CG120). NICE, 2011.

15 Lingford-Hughes AR, Welch S, Peters L, Nutt DJ. BAP updated guidelines: evidence-based guidelines for the pharmacological management of substance abuse, harmful use, addiction and comorbidity: recommendations from BAP. J Psychopharmacol 2012; 26: 899-952.

16 Dorus W, Ostrow DG, Anton R, Cushman P, Collins JF, Schaefer M, et al. Lithium treatment of depressed and nondepressed alcoholics. JAMA 1989; 262: $1646-52$.

17 Salloum IM, Cornelius JR, Daley DC, Kirisci L, Himmelhoch JM, Thase ME. Efficacy of valproate maintenance in patients with bipolar disorder and alcoholism: a double-blind placebo-controlled study. Arch Gen Psychiatry 2005; 62: 37-45.

18 Kemp DE, Gao K, Ganocy SJ, Elhaj O, Bilali SR, Conroy C, et al. A 6-month, double-blind, maintenance trial of lithium monotherapy versus the combination of lithium and divalproex for rapid-cycling bipolar disorder and co-occurring substance abuse or dependence. J Clin Psychiatry 2009; 70 113-21.

19 Brown ES, Sunderajan P, Hu LT, Sowell SM, Carmody TJ. A randomized, double-blind, placebo-controlled, trial of lamotrigine therapy in bipolar disorder, depressed or mixed phase and cocaine dependence. Neuropsychopharmacology 2012; 37: 2347-54.

20 Hollander E, Pallanti S, Allen A, Sood E, Baldini Rossi N. Does sustainedrelease lithium reduce impulsive gambling and affective instability versus placebo in pathological gamblers with bipolar spectrum disorders? Am J Psychiatry 2005; 162: 137-45.

21 Brown ES, Davila D, Nakamura A, Carmody TJ, Rush AJ, Lo A, et al. A randomized, double-blind, placebo-controlled trial of quetiapine in patients with bipolar disorder, mixed or depressed phase, and alcohol dependence. Alcohol Clin Exp Res 2014; 38: 2113-8.

22 Stedman $M$, Pettinati HM, Brown ES, Kotz M, Calabrese JR, Raines S. A double-blind, placebo-controlled study with quetiapine as adjunct therapy with lithium or divalproex in bipolar I patients with coexisting alcohol dependence. Alcohol Clin Exp Res 2010; 34: 1822-31.

23 Tolliver BK, Desantis SM, Brown DG, Prisciandaro JJ, Brady KT. A randomized, double-blind, placebo-controlled clinical trial of acamprosate in alcoholdependent individuals with bipolar disorder: a preliminary report. Bipolar Disord 2012; 14: 54-63.

24 Brown ES, Carmody TJ, Schmitz JM, Caetano R, Adinoff B, Swann AC, et al. A randomized, double-blind, placebo-controlled pilot study of naltrexone in outpatients with bipolar disorder and alcohol dependence. Alcohol Clin Exp Res 2009; 33: 1863-9. 